\title{
Docking Adenosine Receptor Ligands to SARS-CoV2 mRNA Cap Guanine-N7 Methyltransferase
}

\author{
David A. Snyder ${ }^{1, *}$ and Shweta Mehta ${ }^{1}$
}

${ }^{1}$ Department of Chemistry, College of Science and Health, William Paterson University of NJ

*Corresponding author. E-mail: snyderd@wpunj.edu. ORCiD: 0000-0001-6608-2975

\begin{abstract}
The identification of an adenosine binding pocket in the SARS-CoV2 mRNA cap guanine-N7 methyltransferase (nsp16) represents a promising lead in the development of new drugs to combat the devastating pandemic, Covid-19. There are therapeutic agents and drug-like molecules that bind adenosine receptors and hence are likely to bind other adenosine binding proteins such as SARS-CoV2 nsp16. This study explores the docking of known adenosine receptor ligands to SARS-CoV2 nsp16. Among the compounds successfully docked to SARS-CoV2 nsp16 is the anti-inflammatory compound Piclidenoson, which is already in clinical trials for treatment of Covid-19. Energy minimization of the Piclidenoson/nsp16 complex suggests that Piclidenoson binding can induce conformational changes to nsp16.
\end{abstract}

Keywords: SARS-CoV2 mRNA cap guanine-N7 methyltransferase, nsp16, Covid-19, docking, adenosine receptor ligands 


\section{Introduction}

Coronaviruses cause a wide variety of diseases ranging from mild colds and gastrointestinal upset to severe respiratory syndromes including Severe Acute Respiratory Syndrome (SARS) and Middle Eastern Respiratory Syndrome (MERS). ${ }^{1,2}$ The SARS-CoV2 virus is unique among coronaviruses in that it is both highly transmissible and the disease it causes, Covid-19, has high morbidity and mortality rates. ${ }^{3}$ Not surprisingly, the SARS-Cov2 virus has several properties that are unique among coronaviruses.

Like many viruses, coronavirus genomes encode enzymes that cap and otherwise modify viral RNA so that it resembles host mRNA. In particular, coronavirus genomes encode a mRNA cap guanine-N7 methyltransferase, nsp16 (in this paper, nsp16 will refer to SARS-CoV2 nsp16 unless otherwise specified), that modifies capped, viral RNA. The SARS-CoV2 mRNA cap guanine-N7 methyltransferase is unique in that it allosterically binds adenosine. ${ }^{4}$ While the in vivo significance of this site is not yet known, this site is an attractive target for antiviral therapies. One reason this site is an attractive target is that, since adenosine functions as a neurotransmitter with well characterized receptors, many available pharmaceuticals and pharmaceutical lead compounds are known to bind adenosine binding proteins. ${ }^{5,6}$ For instance, one of the most widely used drugs, caffeine, binds adenosine receptors. ${ }^{6}$

This paper reports the results of docking 35 compounds - known adenosine receptor binders, adenosine derivatives and $\beta$-D-fructopyranose - to a crystal structure of the SARS-CoV2 mRNA cap guanine-N7 methyltransferase (PDB ID 6W4H) with $\beta$-D-fructopyranose ${ }^{7}$ in the site which Viswanathan, et al., identified as binding adenosine. ${ }^{4} 12$ compounds, with known affinity for adenosine receptors, are predicted to bind the adenosine binding site in nsp16 better than does adenosine itself. Energy minimized structures for ligand/nsp16 complexes suggest that some of the putative ligands identified in this docking study can perturb nsp16 structure.

\section{Methods}

Identification of Putative Ligands and Docking to Nsp16

Searching adenosine receptor binders in the ZINC database ${ }^{8}$ identified putative nsp16 binders. Putative ligands selected for this docking study included the top 4 highest affinity substances and the top 4 "drugs substances" for each adenosine receptor subtype (ADORA1, ADORA2A, ADORA2B and ADORA3): due to the same substance being present on multiple "top 4" lists, this process gleaned only 27 compounds. Filling out the list of putative ligands were three well known adenosine receptor binders (caffeine, D-limonene and lactucin), one compound similar to one of those established binders (intybin, also known as lactucopicrin, which is a lactucin derivative), three adenosine nucleotides (CAMP, AMP and ATP) and $\beta$-D-fructopyranose (BDF). 
Docking was performed on chain A (nsp16) obtained from a high resolution (1.80 $⿱$ ) structure of the nsp16/nsp10 complex (PDB ID 6W4H) using iGEMDOCK v2.1. ${ }^{9}$ Both unrestricted docking and docking to the region around (binding site radius $10 \AA$ ) the $\beta$-D-fructopyranose (BDF) molecule bound to nsp16 (chain $A$ in $6 \mathrm{~W} 4 \mathrm{H}$ ).

\section{Energy Minimization}

Energy minimizations were performed on the docked adenosine receptor binder/protein complexes with iGEMDOCK binding energies at least as negative as that for adenosine. Each complex consisted of the docked ligand, chain A (nsp16) from $6 \mathrm{~W} 4 \mathrm{H}$ and all ligands bound to chain $A$ (except for BDF, which is in the adenosine binding pocket). In order to evaluate comparable structures, the crystal structure $6 \mathrm{~W} 4 \mathrm{H}$ was also subjected to energy minimization; two systems were considered: one with both chains and all ligands present in the crystal structure and another with only chain A (nsp16) and with the $\beta$-D-fructopyranose ligand removed. The Schrödinger Maestro, version 11.0,10 Protein Prepwizard performed initial preparation of the protein/ligand complexes using default settings except for converting selenomethionine residues to methionines and not creating disulfide bonds. This study only considered atoms with alternate conformations in their maximally occupied positions. A pH of 7.5 was used to adjust protonation states.

The system to be energy minimized included a minimum volume of TIP4PEW water (using a orthorhombic box with a buffer distance of $10 \AA$ on each side), $\mathrm{Na}^{+}$or $\mathrm{Cl}^{-}$ions for neutralization and $0.15 \mathrm{M} \mathrm{NaCl}$. Minimization used default parameters in DESMOND v. $4.8,{ }^{11}$ ran within the Schrödinger Maestro interface.

\section{Analysis of Energy Minimized Structures}

The MatchAlign tool in UCSF Chimera ${ }^{12}$ superimposed energy minimized structures for the $6 \mathrm{~W} 4 \mathrm{H}$ chain A structure without BDF (6W4H $\triangle B D F)$ as well as the structures with docked, putative ligand, onto the energy minimized $6 \mathrm{~W} 4 \mathrm{H}$ structure. Use of the Match->Align tool facilitated extraction (via a header in the displayed sequence alignment) extraction of RMSD values (calculated for all heavy atoms on a per-residue basis) for each superimposition. UCSF Chimera was also used to produce cartoons of protein structures. Both Excel and MATLAB ${ }^{13}$ proved invaluable in collating and plotting RMSD values. 


\section{Results and Discussion}

\section{Docking}

iGEMDOCK indicated that 15 ligands bound at or near the putative adenosine binding site as well as adenosine itself (Table 1, Supplementary Table 1). Those ligands include ATP, AMP and the natural product intybin, a derivative of the adenosine receptor binding natural product lactucin. Also included were the drug Prazosin and a drug candidate compound Piclidenoson (also known as CF-101 and IB-MECA).

Both Prazosin ${ }^{14}$ and Piclidenoson ${ }^{1,15}$ have anti-inflammatory effects. In fact, Konig, et al., have already suggested the use of Prazosin in treating Covid-19 associated cytokine storms ${ }^{14}$, and Piclidenoson is already in Phase-2 clinical trials for use in treating Covid-19. ${ }^{16-18}$ Should either drug prove useful in inhibiting SARS-CoV2 mRNA cap guanine-N7 methyltransferase, they will have two beneficial effects in treating Covid-19: directly inhibiting the viral lifecycle and providing symptomatic relief of disease associated inflammation. Similarly, Intybin has known analgesic effects. $^{19}$ Should it prove useful in inhibiting SARS-CoV2 mRNA cap guanine-N7 methyltransferase, it will also have two beneficial effects in treating Covid-19.

Table 1: Best nsp16 Binders as Ranked by iGEMDOCK Binding Energy

\begin{tabular}{|l|l|l|}
\hline Ligand ID & Name & Binding "Energy" \\
\hline 3924085 & & -127.865 \\
\hline 49872226 & & -114.615 \\
\hline 71332259 & & -113.749 \\
\hline 4261765 & ATP & -110.042 \\
\hline 26014679 & & -109.926 \\
\hline 26015531 & & -109.101 \\
\hline 95616601 & Prazosin & -107.192 \\
\hline 49877703 & & -103.369 \\
\hline 103269598 & & -103.190 \\
\hline 13475415 & & -102.223 \\
\hline 3811810 & Piclidenoson & -102.108 \\
\hline 42888212 & & -98.4758 \\
\hline 2516024 & & -95.9594 \\
\hline 898598 & Intybin & -95.3088 \\
\hline 3860156 & AMP & -94.5175 \\
\hline 2169830 & Adenosine & -92.9955 \\
\hline
\end{tabular}


The best ranked binder identified in this study is ZINC ID 3924085. This compound is not only known to bind adenosine receptors but is also predicted, according to the SEA ${ }^{20}$ predictions in its ZINC database entry, to bind two other methyltransferase enzymes. It may be that SARS-CoV2 mRNA cap guanine-N7 methyltransferase is not the only adenosine binding methyltransferase; or, more generally, it may be that there is a correlation between binding at adenosine binding sites and ability to bind methyltransferase enzymes.

\section{Analysis of Energy Minimized Structures}

While neither the $6 \mathrm{~W} 4 \mathrm{H} \triangle \mathrm{BDF}$ structure nor many of the energy minimized ligand/protein complexes showed much deviation from the energy minimized version of PDB ID 6W4H, four structures showed moderate deviations (e.g. median per-residue, calculated using all heavy atoms, RMSD > $0.15 \AA$, Figure 1). One of those four ligand/protein complexes is the complex of nsp16 with Piclidenoson. Piclidenoson has already been identified (vide supra) as a potential therapeutic agent in Covid-19 treatment, presumably based on its anti-inflammatory properties, but this study suggests it may also exert a therapeutic effect via nsp16 inhibition.

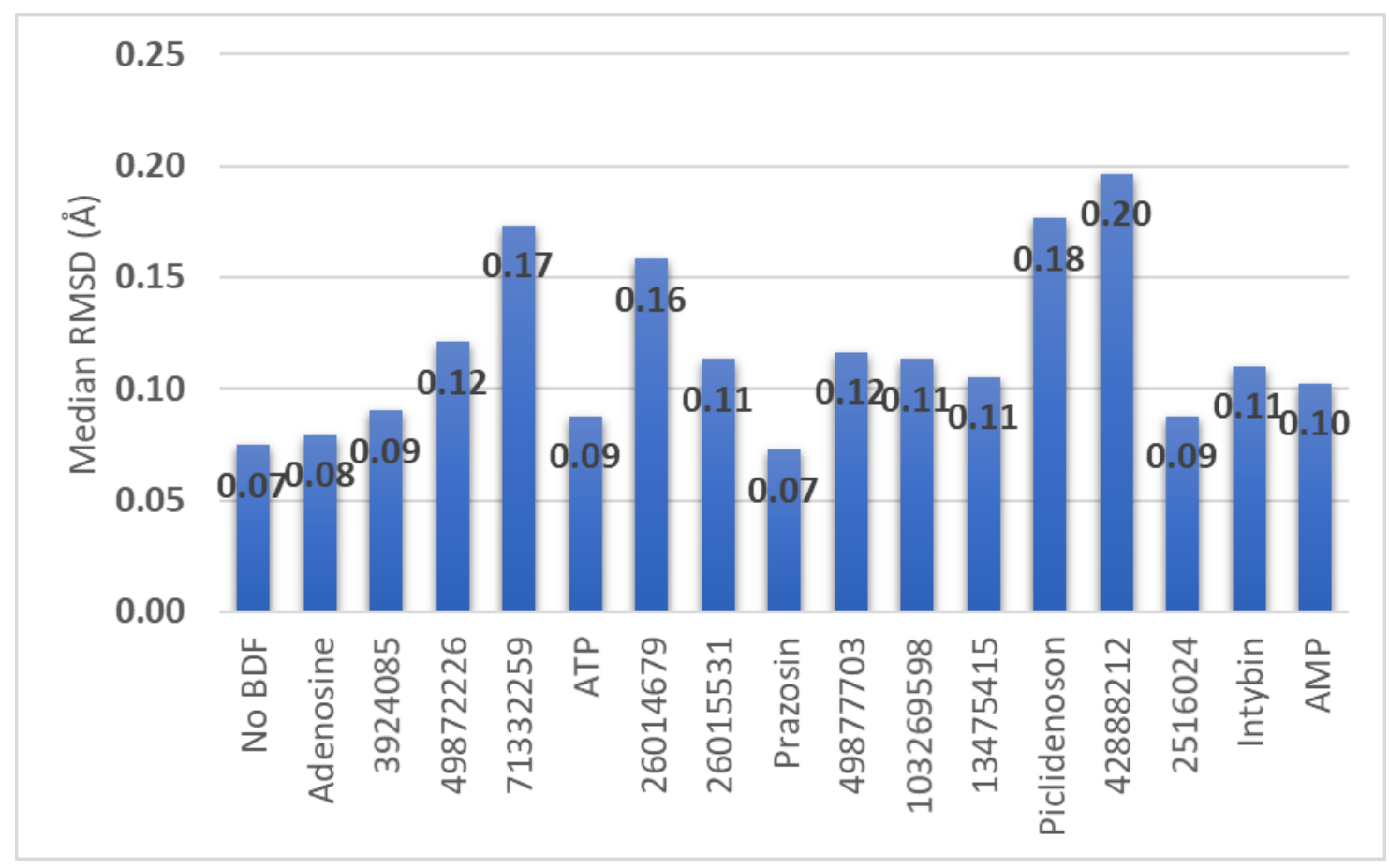

Figure 1: Median per-residue, heavy atom RMSD values from superimpositions of protein/ligand complexes (labeled by either the ligand name or ZINC accession number).

Each of the four ligand/protein complexes, whose energy minimized structures greatly (median per-residue RMSD $>0.15 \AA$ ) deviated from the energy minimized $6 \mathrm{~W} 4 \mathrm{H}$ structure, displayed large scale conformational changes (per residue RMSD > $0.5 \AA$ ) in the nsp16/nsp10 interface (Figure 2). In particular, the energy minimized ZINC ID $71332259 / 6 \mathrm{~W} 4 \mathrm{H}$ chain A complex (Figure 2C) has extensive regions of deviation from the energy minimized $6 \mathrm{~W} 4 \mathrm{H}$ structure in the region of the 
nsp16/nsp10 interface, which is not surprising given the ligand binding site in this complex is nearer to the nsp16/nsp10 interface than is the adenosine binding site identified by Viswanathan, et $a .^{4}$ In the energy minimized structures shown in Figure 2, all but the ZINC ID 42888212 complex (Figure 2A), which actually has the greatest median per-residue RMSD from the energy minimized $6 \mathrm{~W} 4 \mathrm{H}$ structure, have ligands with only a small fraction of binding occurring in the adenosine binding site identified by Viswanathan, et al.

A

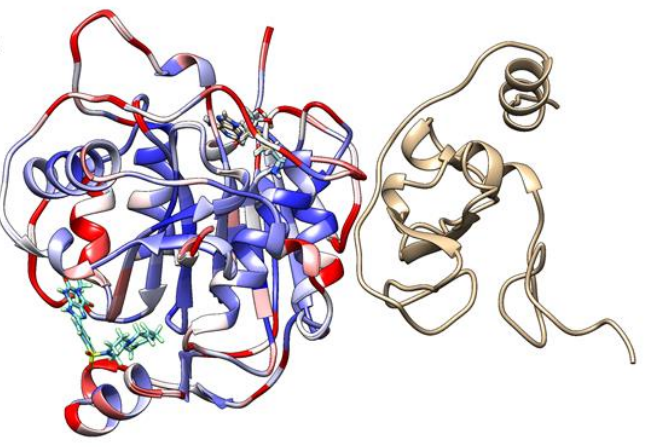

C

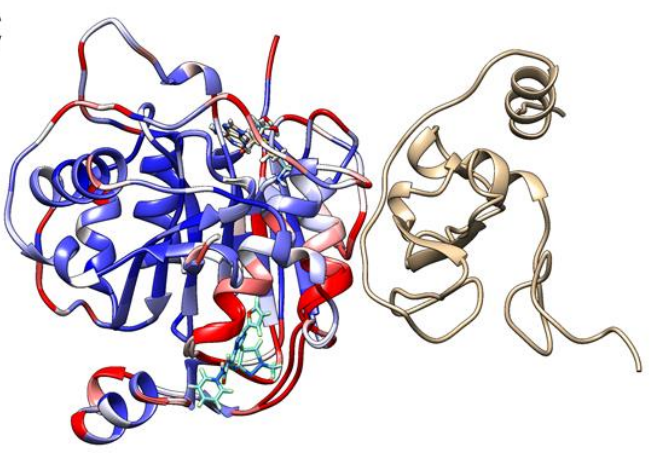

B

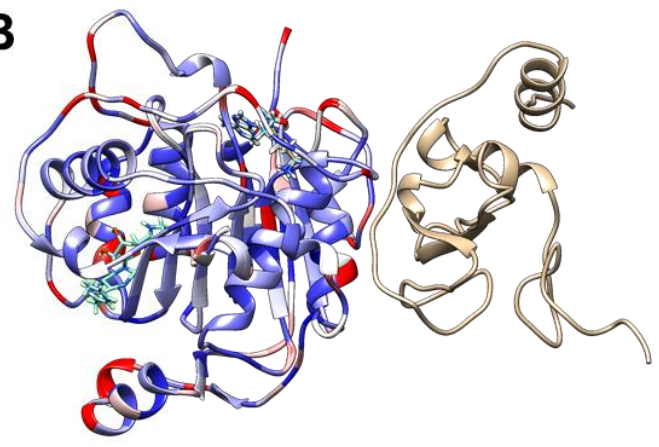

D

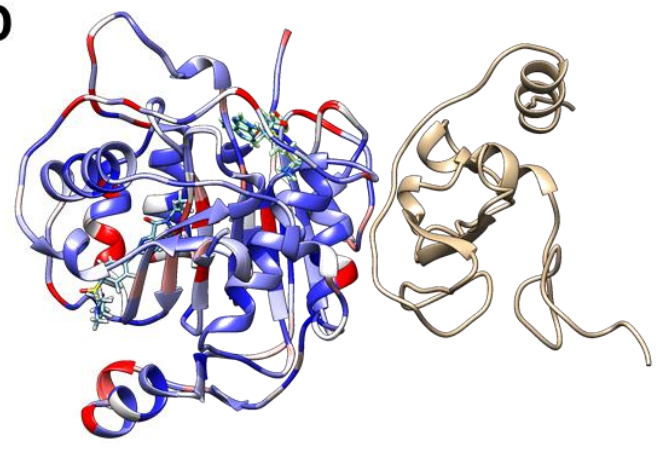

Figure 2: Superimpositions of energy minimized protein ligand complexes and the energy minimized $6 \mathrm{~W} 4 \mathrm{H}$ structure. In each panel, nsp16 coloring tracks the per-residue, heavy atom RMSD between the superimposed structures with red indicating RMSD $>0.5 \AA$, white indicating RMSD $=0.25 \AA$ and blue indicating low RMSD values. Ligands (both the docked ligand and SAdenosyl-Methionine) are rendered as ball and stick models: the adenosine binding site identified by Viswanathan, et al. ${ }^{4}$ is in the lower left corner of each panel (where the ligand is bound in panel A). Complexes, identified by the ZINC ID of the ligand or by name where such a name exists, shown are those with maximal median per-residue RMSD values as plotted in Figure 1: (A) 42888212, (B) Piclidenoson (ZINC ID 3811810), (C) 71332259, and (D) 26014679. 
Given that adenosine receptors are an important target in the treatment of inflammatory diseases ${ }^{5,15}$ and the role that an over-active immune response plays in the pathogenesis of Covid$19,{ }^{14}$ the identification of an adenosine binding pocket in a critical SARS-CoV2 protein ${ }^{4}$ suggests the possibility of finding drugs that target both SARS-CoV2 induced inflammatory responses and the reproduction of the SARS-CoV2 virus itself. This study identified multiple adenosine receptor binders likely to also bind at or near the SARS-CoV2 mRNA cap guanine-N7 methyltransferase (nsp16) adenosine binding pocket.

Among those compounds identified are two molecules already being studied for treatment of Covid-19. Another molecule (ZINC ID 71332259), binding near the nsp16 adenosine binding pocket, potentially (as judged by changes in energy minimized structures with the ligand bound vs. with $\beta$-D-fructopyranose bound) can disrupt the nsp16/nsp10 interface necessary for SARSCoV2 replication. ${ }^{21}$ Since this molecule docks at the edge of the adenosine binding pocket unique to SARS-CoV2 nsp16, it may prove useful in disrupting nsp16/nsp10 interactions in multiple coronavirus species. The identification of a molecule hypothetically interfering with protein/protein interactions by docking to an allosteric site suggests that docking, combined with energy minimization, is a useful tool for identifying molecules that potentially interfere with protein/protein interactions.

\section{Acknowledgements}

The authors thank James Aramini for presenting a pre-print of the paper by Viswanathan, et al., ${ }^{4}$ whose identification of an adenosine binding site on a critical SARS-Cov2 protein inspired the authors to conduct this study, to the Rensselaer Polytechnic Institute COVID19 Journal Club. The authors also thank Gaetano Montelione for arranging this journal club. An award (to D.A.S.) of Assigned Release Time (ART) for research from the Office of the Provost of William Paterson University of NJ facilitated completion of this work.

\section{Declaration of Interests}

The authors have no conflicts of interest to declare.

\section{Funding Details}

The authors have received no external funding to conduct this research study.

\section{Data Availability}

The energy minimized complexes between proteins and docked ligands are available from the corresponding author upon request. 


\section{Supplemental Data}

Supplementary Table 1: Compounds Studied in this Docking Study. Receptor binding for nucleotides is not indicated as those compounds (blue) were not selected based on (potential) adenosine receptor binding. Sources of information about which receptors the given ligand binds are (a) CHemBL 20 (as indicated in the compound's page in the ZINC database), (b) the compound's page on CHEBI and (c) the compound's page on PUBMED. The justifications for including the compound in this study are that it is indicated in the ZINC database as being one of the top 4 adenosine receptor - (i) 1 (ADORA1), (iia) 2A (ADORA2A), (iib) 2B (ADORA2B) and (iii) 3 (ADORA3) - binders, being one of the top 4 adenosine receptor - (I) 1 (ADORA1), (Ila) 2A (ADORA2A), (IIb) 2B (ADORA2B) and (III) 3 (ADORA3) - binding drugs, (g) based on general knowledge, $(\mathrm{n})$ that it is an adenosine nucleotide and $(p)$ it is present in a crystal structure in the binding pocket being probed.

\begin{tabular}{|c|c|c|c|c|c|c|}
\hline \multirow[b]{2}{*}{ Ligand ID } & \multirow[b]{2}{*}{ Name } & \multicolumn{5}{|c|}{ Binding "Energy" from iGEMDOCK } \\
\hline & & BDF Site & Unconstrained & Receptors & Source & $\underline{\text { Rationale }}$ \\
\hline 3924085 & & -127.865 & -132.176 & ADORA1, ADORA2A & (a) & (iia) \\
\hline 49872226 & & -114.615 & -118.832 & ADORA1, ADORA2A, ADORA2B & (a) & (iib) \\
\hline 71332259 & & -113.749 & -136.032 & ADORA3 & (a) & (iii) \\
\hline 4261765 & ATP & -110.042 & -142.907 & $\mathrm{~N} / \mathrm{A}$ & $\mathrm{N} / \mathrm{A}$ & (n) \\
\hline 26014679 & & -109.926 & -113.676 & ADORA1 & (a) & (i) \\
\hline 26015531 & & -109.101 & -107.098 & ADORA1 & (a) & (i) \\
\hline 95616601 & Prazosin & -107.192 & -120.318 & ADORA3, etc. & (a) & (iii) \\
\hline 49877703 & & -103.369 & -127.484 & ADORA2A, ADORA2B & (a) & (iib) \\
\hline 103269598 & & -103.19 & -118.902 & ADORA1, ADORA2A, ADORA2B, ADORA3 & (a) & (iib) \\
\hline 13475415 & & -102.223 & -129.190 & ADORA1, ADORA2A, ADORA2B, ADORA3 & (a) & (iii) \\
\hline 3811810 & Piclidenoson & -102.108 & -105.614 & ADORA1, ADORA2A, ADORA2B, ADORA3 & (a) & (iii) \\
\hline 42888212 & & -98.4758 & -119.854 & ADORA1, ADORA2A, ADORA2B & (a) & (iib) \\
\hline 2516024 & & -95.9594 & -93.7110 & ADORA1, ADORA2A & (a) & (i) \\
\hline 898598 & Intybin & -95.3088 & -112.137 & $?$ & (b) & (g) \\
\hline 3860156 & AMP & -94.5175 & -112.766 & $\mathrm{~N} / \mathrm{A}$ & $\mathrm{N} / \mathrm{A}$ & (n) \\
\hline 2169830 & Adenosine & -92.9955 & -98.9584 & ADORA1, ADORA2A, ADORA3, etc. & (a) & (p),(i),(iia) \\
\hline 49784476 & & -92.3989 & -121.047 & ADORA1, ADORA2A, ADORA2B, ADORA3 & (a) & (iii) \\
\hline 3873977 & cAMP & -91.5504 & -101.337 & $\mathrm{~N} / \mathrm{A}$ & $\mathrm{N} / \mathrm{A}$ & (n) \\
\hline 4216238 & & -87.1933 & -88.8634 & ADORA1, ADORA2A & (a) & (i),(iia) \\
\hline 49072969 & & -81.4281 & -98.5377 & ADORA1, ADORA2A & (a) & (i) \\
\hline 1530776 & Eht0201 & -81.397 & -97.7419 & ACHE, ADORA2B & (a) & (IIB) \\
\hline 19144216 & Pyrilamine & -79.567 & -94.4813 & ADORA3, etc. & (a) & (III) \\
\hline 3814423 & Cyproterone & -79.4722 & -105.083 & ADORA1, etc. & (a) & (I) \\
\hline 96913999 & & -79.2381 & -83.9763 & ADORA2A & (a) & (iia) \\
\hline 49069606 & & -78.1205 & -97.6733 & ADORA1, ADORA2A & (a) & (iia) \\
\hline 96914006 & & -77.9239 & -80.8353 & ADORA2A & (a) & (iia) \\
\hline 897089 & Mefloquin & -74.2772 & -75.2000 & ADORA1, ADORA2A, ADORA3, KCNH2 & (a) & $(I I A),(I I B)$ \\
\hline 18043251 & Theophylline & -74.0092 & -74.4111 & ADORA1, ADORA2A, ADORA2B, ADORA3 & (a) & $(I),(I I A),(I I B)$ \\
\hline 403567 & Enprofylline & -73.9011 & -83.7338 & ADORA2A, ADORA2B & (a) & (IIB) \\
\hline
\end{tabular}




\begin{tabular}{|c|c|c|c|c|c|c|}
\hline 389747 & Naloxone & -72.0337 & -88.3865 & ADORA3, etc. & (a) & (III) \\
\hline 519270 & Lactucin & -71.1499 & -92.4700 & Unidentified Adenosine Receptor(s) & (b) & (g) \\
\hline 1530764 & $\begin{array}{l}\text { Protriptyline } \\
\beta \text {-D- }\end{array}$ & -69.7285 & -86.1253 & ADORA3, SLC6A2 & (a) & (III) \\
\hline 1532739 & fructopyranose & -66.1619 & -74.5833 & N/A & $\mathrm{N} / \mathrm{A}$ & (p) \\
\hline 1084 & Caffeine & -65.2476 & -78.3291 & ADORA2A, etc. & (a) & (g) \\
\hline 968226 & D-Limonene & -48.5445 & -54.5227 & ADORA2A & (c) & (g) \\
\hline
\end{tabular}

\section{References}

1. Nascimento Junior, José Adão Carvalho, Santos AM, Quintans-Júnior LJ, Walker CIB, Borges LP, Serafini MR. SARS, MERS and SARS-CoV-2 (COVID-19) treatment: A patent review. Expert Opinion on Therapeutic Patents 2020(just-accepted).

2. Amirian ES, Levy JK. Current knowledge about the antivirals remdesivir (GS-5734) and GS-441524 as therapeutic options for coronaviruses. One Health 2020:100128.

3. Peeri NC, Shrestha N, Rahman MS, Zaki R, Tan Z, Bibi S, Baghbanzadeh M, Aghamohammadi N, Zhang W, Haque U. The SARS, MERS and novel coronavirus (COVID-19) epidemics, the newest and biggest global health threats: What lessons have we learned? Int J Epidemiol 2020.

4. Viswanathan T, Arya S, Chan S, Qi S, Dai N, Hromas RA, Park J, Oladunni F, MartinezSobrido L, Gupta YK. Structural basis of RNA cap modification by SARS-CoV-2 coronavirus. bioRxiv 2020.

5. Kaiser SM, Quinn RJ. Adenosine receptors as potential therapeutic targets. Drug Discov Today 1999;4(12):542-51.

6. Rivera-Oliver M, Díaz-Ríos M. Using caffeine and other adenosine receptor antagonists and agonists as therapeutic tools against neurodegenerative diseases: A review. Life Sci 2014;101(1-2):1-9.

7. Lemus MR, Minasov G, Shuvalova L, Inniss NL, Kiryukhina O, Wiersum G, Kim Y, Jedrzejczak R, Maltseva NI, Endres M. The crystal structure of nsp10-nsp16 heterodimer from SARS CoV-2 in complex with S-adenosylmethionine. bioRxiv 2020.

8. Irwin JJ, Shoichet BK. ZINC- a free database of commercially available compounds for virtual screening. Journal of Chemical Information and Modeling 2005;45(1):177-82.

9. Hsu K, Chen Y, Lin S, Yang J. iGEMDOCK: A graphical environment of enhancing GEMDOCK using pharmacological interactions and post-screening analysis. BMC Bioinformatics 2011;12(1):S33. 
10. Schrodinger L. Schrodinger software suite. New York: Schrödinger, LLC 2011;670.

11. AnonymousScalable algorithms for molecular dynamics simulations on commodity clusters. SC 2006 conference, proceedings of the ACM/IEEEIEEE; 2006. 43 p.

12. Pettersen EF, Goddard TD, Huang CC, Couch GS, Greenblatt DM, Meng EC, Ferrin TE. UCSF chimera-a visualization system for exploratory research and analysis. Journal of Computational Chemistry 2004;25(13):1605-12.

13. The MathWorks I. MATLAB [computer program]. Natick, Massachusetts, United States: .

14. Konig MF, Powell MA, Staedtke V, Bai R, Thomas DL, Fischer NM, Huq S, Khalafallah AM, Koenecke A, Xiong R. Preventing cytokine storm syndrome in COVID-19 using $\alpha-1$ adrenergic receptor antagonists. J Clin Invest 2020.

15. Cohen S, Fishman P. Targeting the A3 adenosine receptor to treat cytokine release syndrome in cancer immunotherapy. Drug Design, Development and Therapy 2019;13:491.

16. [Anonymous]2020 Piclidenoson for treatment of COVID-19. $<$ https://clinicaltrials.gov/ct2/show/NCT04333472>. Accessed 202026 May.

17. Shweta F, Murugadoss K, Awasthi S, Venkatakrishnan AJ, Puranik A, Kang M, Pickering BW, O'Horo JC, Bauer PR, Razonable RR. Augmented curation of unstructured clinical notes from a massive EHR system reveals specific phenotypic signature of impending COVID-19 diagnosis. arXiv Preprint arXiv:2004.093382020.

18. Paumgartten FJR, Delgado IF, da Rocha Pitta L, de Oliveira, Ana Cecilia Amado Xavier. Drug repurposing clinical trials in the search for life-saving covid-19 therapies; research targets and methodological and ethical issues. Vigilância Sanitária Em Debate: Sociedade, Ciência \& Tecnologia 2020.

19. Wesołowska A, Nikiforuk A, Michalska K, Kisiel W, Chojnacka-Wójcik E. Analgesic and sedative activities of lactucin and some lactucin-like guaianolides in mice. J Ethnopharmacol 2006;107(2):254-8.

20. Keiser MJ, Roth BL, Armbruster BN, Ernsberger P, Irwin JJ, Shoichet BK. Relating protein pharmacology by ligand chemistry. Nat Biotechnol 2007;25(2):197-206.

21. Chen Y, Su C, Ke M, Jin X, Xu L, Zhang Z, Wu A, Sun Y, Yang Z, Tien P. Biochemical and structural insights into the mechanisms of SARS coronavirus RNA ribose 2'-0methylation by nsp16/nsp10 protein complex. PLoS Pathogens 2011;7(10). 